\title{
THE REPRODUCTION OF PARAMAECIUM AURELIA IN A 'CONSTANT' CULTURE MEDIUM OF BEEF EXTRACT
}

\author{
LORANDE LOSS WOODRUFF AND GEORGE ALFRED BAITSELL \\ Sheffield Biological Laboratory, Yale University
}

TWO FIGURES

Previous work ${ }^{1}$ with pedigree cultures of Paramaecium aurelia and Paramaecium caudatum has apparently shown that the life history of these forms, when bred continuously on infusions of hay made up exactly the same from day to day, tends to run in a cycle which terminates with the death of the culture. Previous work has also shown that Paramaecium aurelia ${ }^{2}$ may be bred indefinitely on a culture medium which is frequently varied.

In view of these results the following question suggests itself: Is the longevity of Paramaecium on a 'varied environment' dependent upon intrinsic stimuli from the frequent changes of the medium, or is a 'constant' medium of hay infusion uniavorable because it lacks some elements which are essential for the continued existence of this protozoön?

To test this point it is necessary to find, if possible, a suitable 'constant' culture medium which contains all the elements which the organism demands, and to determine its effect on the vitality of Paramaecium when subjected to it for a considerable length of time. If such a suitable medium is secured on which paramaecia will live indefinitely, it is apparent that the possible continual daily stimulation afforded by 'varied' culture media is

${ }^{1}$ Calkins: Jour. Exp. Zoöl., vol. 1, no. 3, 1904 . Woodruff: Biol. Bull., vol. 17, no. 4, 1909 .

${ }^{2}$ L. L. Woodruff: Two thousand generations of Paramaecium. Archiv fi. Protistenkunde, Bd. 21, 3, 1911. 
not the crucial factor in the determination of the longevity of paramaecia cultures. Further, and aside from this interesting theoretical consideration, such a favorable 'constant' culture medium would be valuable for breeding paramaecia in various lines of experimental work, since it is clear from many investigations that the reactions of paramaecia to various reagents, etc., are greatly modified by their past and present environment.s

In the present paper are briefly outlined the results which have been secured, thus far, in an effort to answer the question suggested above, and to provide a suitable culture medium which investigators can employ in breeding cultures of this organism.

The animals used in this study were from the pedigree culture of Paramaecium aurelia which one of $\mathrm{us}^{4}$ has had under daily observation for more than four years, and which has attained, up to the present time (May 1, 1911), 2370 generations under the conditions of a 'varied environment,' without conjugation or artificial stimulation.

The favorable results secured first by Calkins ${ }^{5}$ with strong solutions of beef extract as a temporary stimulant for his degenerating cultures of Paramaecium caudatum in infusions of hay, and later by Woodruffo with Oxytricha fallax under similar conditions, suggested the use of a weak extract of beef as a 'constant' culture medium. Further, beef extract should afford all the elements required for the continued life of protoplasm. The results of chemical analyses have shown that Liebig's extract of beef is

${ }^{3}$ For example, Greeley (Biol. Bull., vol. 6. 1904, p. 1), in a study of the effect of various chemicals on the protoplasm of Paramaecium, wrote: "Maximal dilutions can only be approximate, as the action of identical solutions is not the same on paramaecia from different cultures, because no two are exactly alike in respect to chemical composition and osmotic pressure;" and Miss Towle in a similar study (Amer. Jour. Physiol, vol. 11, no. 2, 1904, p. 235) said: "The first step toward a clearing of the haze that envelops the subject will be found, I believe, when an effort is made to unify the conditions under which different investigators are working."

${ }^{4}$ Woodruff: Loc. eit.

- Calkins: (Archiv für Entwick.-Mechan., Bd. 15, 1,1902). "The lean beef was boiled in tap-water for fifteen minutes and allowed to stand until cool. The clear fluid was then used without dilution."

'Woodruff: Jour. Exp. Zoöl., vol. 2, no. 4, 1905. 
remarkably constant in composition, and therefore this standard preparation, which is available for all investigators, was used as the basis of our culture medium.

Having decided on Liebig's extract of beef, it was necessary to make a series of experiments to determine the strength of solution which was most favorable for Paramaecium. The solutions were made by weighing out one gram of the extract and diluting this with varying amounts of distilled water. The different concentrations of beef extract showed that a solution of approximately 0.025 per cent gave the best results. Accordingly a quantity of this solution was made up which was sufficient to provide culture medium for the organisms for a period of seven months. This length of time was decided upon for this work as the final results of Calkins' experiments led him to conclude that the cycle of Paramaecium caudatum, in a constant environment of hay infusion, was not of more than six months duration. ${ }^{7}$ The medium when made was put into over one hundred test tubes, and these were plugged with cotton and sterilized. The solution in the various tubes remained sterile until it was used, and the inoculation of the medium with bacteria which were transferred with the paramaecia afforded an ample supply of food for the animals.

The regular experiment was begun on October 1, 1910, by the isolation of a specimen from each of the four lines of the pedigree culture I of Paramaecium aurelia at the 2012th generation. Each of the organisms was placed on a depression slide in five drops of the beef extract solution, and in this manner was started a culture designated Paramaecium IB. This culture was continued by isolating an organism every day from each of the four lines of the culture, and placing it in fresh culture medium on a sterile depression slide. The number of divisions during the previous twenty-four hours was recorded at the time of isolation. From this record the average daily rate of division of the four lines

\footnotetext{
${ }^{7}$ It may be noted that animals from this same pedigree culture of $P$. aurelia were bred on a 'constant' medium of hay infusion from February to June, 1909, and died out after 107 days subjection to this condition. (Cf. Biol. Bull., vol. 17, no. 4, 1909, fig. 4).
} 


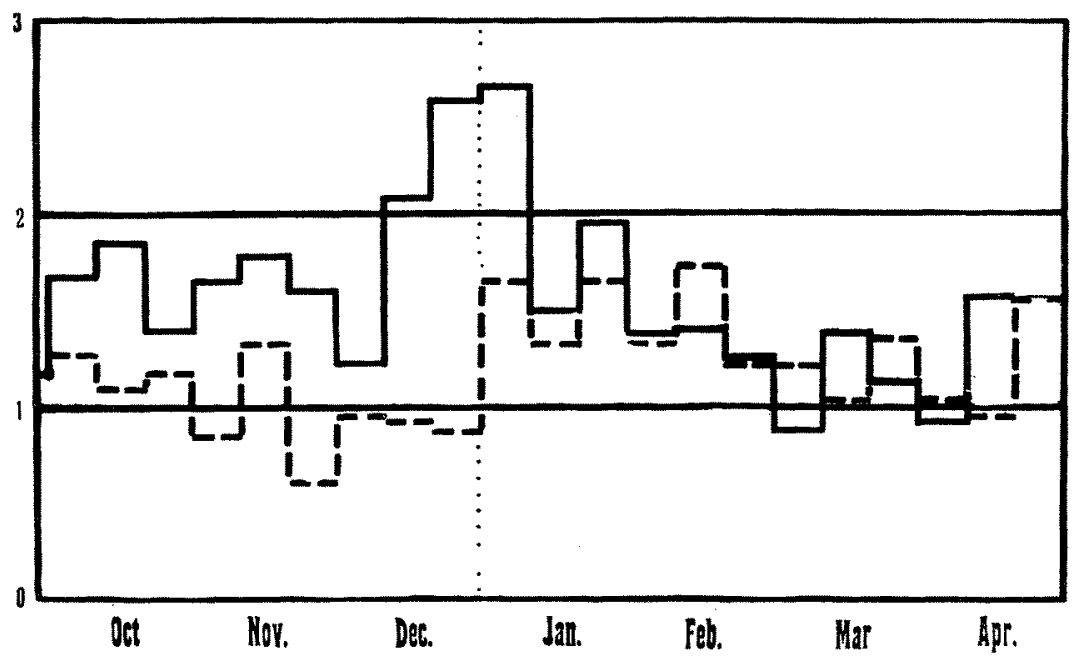

Fig. 1 Graph showing the rate of division of Paramaecium aurelia, Culture I and Culture IB, from October 1, 1910, through April 29, 1911. The ordinates represent the average daily rate of division of the four lines of the respective cultures, again averaged for ten day periods. Culture $I=-$; Culture IB $=-\cdots-$

of the culture, again averaged for five- and ten-day periods, was computed, and the result is graphically shown in figs. 1 and 2 .

The original pedigree culture on a varied environment was, of course, continued, and served as a control for the culture on beef extract, since the method of carrying on the two cultures was identical, except that the medium used was not the same in each case. The original pedigree culture was bred on infusions of grass, hay, pond weeds, etc., made up with water from various sources. The infusions were boiled before being used to prevent the introduction of 'wild' paramaecia into the pure lines. ${ }^{8}$

The various preparations were kept in moist chambers to prevent evaporation, and the temperature of the air in these chambers was recorded by a maximum and minimum registering thermometer. Obviously this method of recording the temperature gives only the extremes to which the cultures were subjected,

s For further details of the method employed, ef., Woodruff, loc. cit. 
and does not take into account the length of time during which any particular temperature was maintained. However, the method is sufficiently exact for the problem at hand. Studies on the relation of temperature to the 'rhythms' in the rate of reproduction of paramaecia are now in progress. During the first three months of the work, culture I ('varied' culture medium) and culture IB (beef) were kept in different rooms, and therefore during this time the cultures were subjected to different temperatures. From January 1 to the present time, both cultures were kept in the same place and consequently each was subjected to the same temperature.

There were, then, two pedigree cultures of Paramaecium, each comprising four lines, being conducted simultaneously. One of these had been bred on a 'varied' culture medium for fortyone months, and was continued under the same conditions during the following seven months, i.e., to the present time. The other culture, isolated line by line from the first culture, was carried on for seven months (to date) on a 'constant environment' of beef extract. The chemical composition of this medium was identical from day to day as it was all made up and sterilized at the same time. The only variation, therefore, in the medium used for these organisms on beef extract was the fluctuations in the bacterial flora due to infections from the air, and slight variations in the multiplication of the bacteria due to temperature changes. This, however, was so small that it is negligible from the standpoint of these experiments.

A study of figs. 1 and 2 gives a clear idea of the comparative rate of division of the two cultures, and shows that, at the end of the seven months work, the rate of division, and therefore presumably the vitality, of the two series of animals is practically the same. Neither of the cultures shows any indications of loss of vigor, and the rate of division of each at the end of the experiment is practically the same as at the beginning-such fluctuations as have occurred being merely 'rhythms'.

During the first three months of the work, the rate of division of the beef series was considerably lower than that of the other series, but this is obviously explained by the considerably higher 


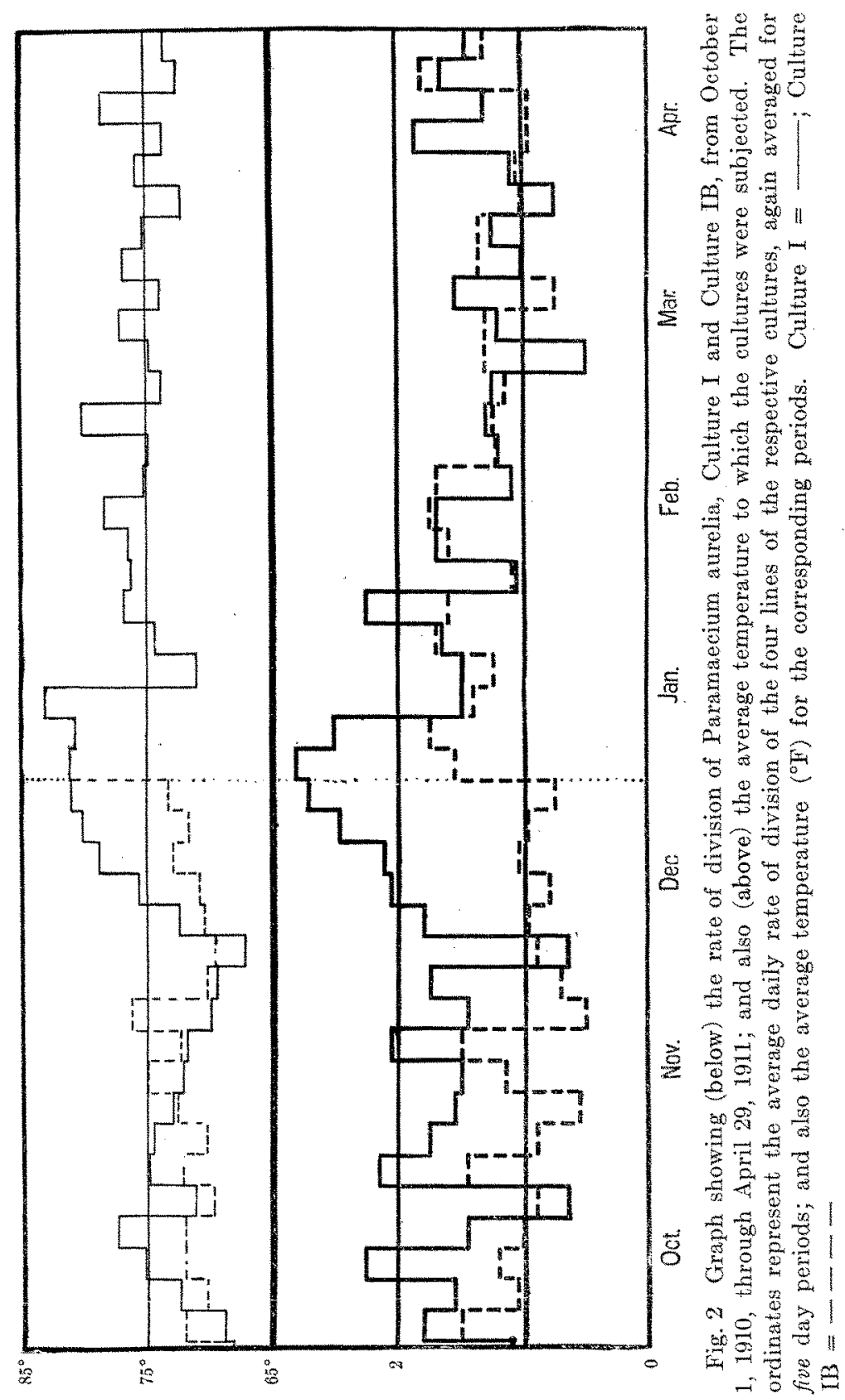


temperature to which the latter was subjected during this period (cf. temperature curves in fig. 2). During this period of three months, culture I advanced from the 2012th to the 2188th generation, or 176 generations, whereas culture IB advanced from the 2012th to the 2120th generation, or 108 generations. A comparison of the rate of division from January 1 to April 29, 1911, when both cultures were subjected to identical temperature conditions, shows that culture I advanced from the 2188th to the 2365th generation, or 177 generations, while culture IB advanced from the 2120th to the 2287th generation, or 167 generations. Therefore the net variation in the number of generations attained by the two cultures during the last four months, when under the same conditions of temperature, was only ten. Further, the appearance and behavior of the paramaecia in the two cultures were identical.

It is evident, then, that the 'constart' medium of beef extract employed has proved (during the seven months of this experiment) to be practically as favorable a medium for the reproduction of this pedigree culture of Paramaecium aurelia as the "varied environment' medium, ${ }^{9}$ and therefore, the conclusion seems justified that this culture of Paramaecium can, in all probability, be continued indefinitely on this 'constant' medium. It therefore appears fair to conclude that it is the 'composition' of the medium rather than the 'changes' in the medium which is conducive to the unlimited development of this culture without conjugation or artificial stimulation.

It is not suggested that every culture of Paramaecium. would have the potential to attain more than two thousand three hundred generations under the conditions of a 'varied environment,' nor is it suggested that every culture of Paramaecium would thrive for over seven months on a 'constant' environment of beef extract. "For undoubtedly there are strong and weak strains

\footnotetext{
"Experiments are now in progress to determine if this culture of Paramaecium will develop indefinitely on infusions of hay which are not made up the same from day to day, i.e., if hay infusion, in which there is a slight daily variation, may not be substituted for the decidedly varied culture medium which has been used during the past four years.
} 
among Infusoria as among other classes of animals. Again, it is possible that the different races of paramaecia which Jennings has been able to isolate may have a physiological as well as a morphological basis of distinction."10 But it is believed that these experiments clearly show that beef extract (in the concentration used) is a suitable environment for the continued reproduction of this pedigree culture of Paramaecium, and that beef extract, in this or closely similar solutions, will prove to be a favorable medium for use in many investigations on the physiology of Paramaecium."

${ }^{10}$ Woodruft: Biol. Bull., vol. 17, no. 4, September, 1909, p. 303.

11 After the completion of the seven months experiment (April 29, 1911), a new lot of the beef extract medium was made up exactly the same as before and the culture has been continued on this without noticeable change in its reproduction to date of correcting proof, June 20, 1911. 